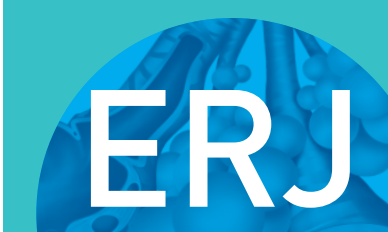

open research
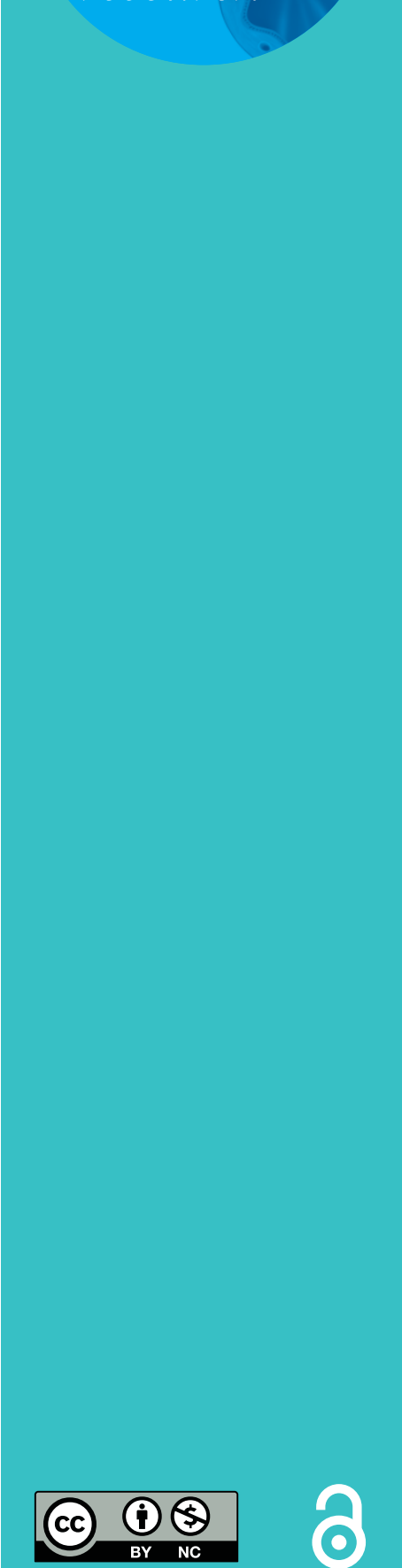

\section{Pneumothoraces in collagen VI-related dystrophy: a case series and recommendations for management}

\author{
Kristin L. Fraser ${ }^{1}$, Scott Wong ${ }^{2}$, A. Reghan Foley ${ }^{3}$, Sameer Chhibber ${ }^{1}$, \\ Carsten G. Bönnemann ${ }^{3}$, Daniel J. Lesser ${ }^{4}$, Carla Grosmann ${ }^{5}$ and \\ Anne Rutkowski ${ }^{6}$
}

Affiliations: ${ }^{1}$ University of Calgary, Calgary, AB, Canada. ${ }^{2}$ The Congenital Muscle Disease International Registry, Torrance, CA, USA. ${ }^{3}$ Neuromuscular and Neurogenetic Disorders of Childhood Section, National Institute of Neurological Disorders and Stroke, NIH, Bethesda, MD, USA. "Division of Respiratory Medicine, Rady Children's Hospital San Diego, University of California San Diego, San Diego, CA, USA. ${ }^{5}$ Division of Neurosciences, University of California San Diego, San Diego, CA, USA. ${ }^{6}$ Cure CMD and Kaiser Permanente, Los Angeles, CA, USA.

Correspondence: Kristin L. Fraser, University of Calgary, 700714 St SW, Calgary Alberta, T2V1P9, Canada. E-mail: kristin.fraserdalbertahealthservices.ca

ABSTRACT Collagen VI-related dystrophy (collagen VI-RD) is a rare neuromuscular condition caused by mutations in the COL6A1, COL6A2 or COL6A3 genes. The phenotypic spectrum includes early-onset Ullrich congenital muscular dystrophy, adult-onset Bethlem myopathy and an intermediate phenotype. The disorder is characterised by distal hyperlaxity and progressive muscle weakness, joint contractures and respiratory insufficiency. Respiratory insufficiency is attributed to chest wall contractures, scoliosis, impaired diaphragmatic function and intercostal muscle weakness. To date, intrinsic parenchymal lung disease has not been implicated in the inevitable respiratory decline of these patients.

This series focuses on pneumothorax, an important but previously under-recognised disease manifestation of collagen VI-RD.

We describe two distinct clinical presentations within collagen VI-RD patients with pneumothorax. The first cohort consists of neonates and children with a single pneumothorax in the setting of large intrathoracic pressure changes. The second group is made up of adult patients with recurrent pneumothoraces, associated with chest computed tomography scan evidence of parenchymal lung disease. We describe treatment challenges in this unique population with respect to expectant observation, tube thoracostomy and open pleurodesis.

Based on this experience, we offer recommendations for early identification of lung disease in collagen VI-RD and definitive intervention.

@ERSpublications

Collagen VI-RD patients may experience unprovoked or recurrent pneumothorax from parenchymal lung changes http://ow.ly/ZL3h30ce0Bk

Cite this article as: Fraser KL, Wong S, Foley AR, et al. Pneumothoraces in collagen VI-related dystrophy: a case series and recommendations for management. ERJ Open Res 2017; 3: 00049-2017 [https://doi.org/10.1183/23120541.00049-2017].

Received: April 102017 | Accepted after revision: May 142017

Conflict of interest: Disclosures can be found alongside this article at openres.ersjournals.com

The content of this work is not subject to copyright. Design and branding are copyright @ERS 2017. This article is open access and distributed under the terms of the Creative Commons Attribution Non-Commercial Licence 4.0. 


\section{Background}

Collagen VI-related dystrophy (collagen VI-RD) is a common form of congenital muscular dystrophy (CMD) resulting from mutation(s) in COL6A1, COL6A2 or COL6A3, the genes encoding for collagen VI, and leading to connective tissue defects and respiratory insufficiency [1]. The collagen VI-RD phenotypic spectrum includes congenital-onset Ullrich congenital muscular dystrophy (UCMD), a milder form that typically manifests during adulthood called Bethlem myopathy and intermediate phenotypes. Collagen VI-RD can be caused by de novo dominant mutations leading to mislocalised abnormal collagen VI within the muscle extracellular matrix or recessive loss-of-function mutations resulting in complete absence of collagen VI. Patients typically present clinically with progressive proximal muscle weakness, distal joint hyperlaxity and proximal joint contractures.

Respiratory impairment in collagen VI-RD has, to date, been attributed to changes in chest wall compliance and diaphragmatic function [2]. A retrospective study of 145 patients with collagen VI-RD confirmed and quantified progressive respiratory insufficiency for both the UCMD and intermediate phenotypes. Those with UCMD demonstrate a lack or loss of ambulation prior to age 10 years, a $2.6 \%$ annual decline in forced vital capacity (FVC) and the need for nocturnal ventilator support at a mean age of 11.3 years. The intermediate phenotype, in contrast, is ambulant through early adult years, with a $2.3 \%$ annual decline in FVC and the initiation of ventilator support at a mean age of 20.7 years. Interestingly, Bethlem myopathy does not show progressive respiratory insufficiency [3].

Collagen VI is a key component of the extracellular matrix in skeletal muscle. It is secreted as tetramers by the muscle interstitial fibroblasts after undergoing an assembly process requiring collagen VI $\alpha 1, \alpha 2$ or $\alpha 3$ chains in equal molarity to form, at first, monomers. In the extracellular space, tetramers assemble to form a microfibrillar matrix. While collagen VI has been shown by immunofluorescence to localise both to the bronchial tree and the alveoli, its function and contribution to lung physiology are not yet known [4]. To date, there has been no evidence to suggest that parenchymal lung involvement contributes to progressive respiratory insufficiency in those with collagen VI-related myopathy. Here, we report on several patients with genetically confirmed collagen VI-RD with single and/or recurrent pneumothoraces. We describe this population as two distinct cohorts based upon age. In the adult cohort, radiographic images demonstrate evidence of lung parenchymal involvement with subpleural blebs.

\section{Cohort 1: neonatal and childhood pneumothoraces associated with hospitalisation}

Patients 1 and 2 are siblings, each of whom developed a pneumothorax on their first day of life. Patient 1 , born at term, developed respiratory distress due to a pneumothorax within hours of uncomplicated vaginal delivery. He required a $24 \mathrm{~h}$ stay in the neonatal intensive care unit (NICU) and was treated with supplemental oxygen. Patient 2 presented with grunting and respiratory distress immediately following term birth by the vaginal route. Bilateral pneumothoraces and pneumomediastinum were associated with chest-radiographic evidence of right clavicle fracture. She required $48 \mathrm{~h}$ of care in the NICU. Both siblings were noted to have bilateral congenital hip dislocations upon discharge from the NICU. Patient 1 was diagnosed with collagen VI-RD of an intermediate phenotype at age 15 years, with two mutations in COL6A2. His sister has the same mutations. To date, neither sibling has had a recurrent pneumothorax, despite the fact that patient 1 required a prolonged period of ventilation post scoliosis surgery at age 16 . Neither sibling is using non-invasive positive pressure ventilation (NIPPV) and both remain ambulant (currently aged 22 years and 18 years).

Patient 3 is a 16 -year-old male with UCMD, homozygous for two mutations in the COL6A3 gene with associated restrictive lung disease and kyphoscoliosis. He presented at age 13 years for posterior spinal fusion. He was on nocturnal NIPPV for 2 years prior to the planned procedure, and pulmonary function testing revealed a FVC of $33 \%$ predicted $(1.09 \mathrm{~L})$, forced expiratory volume in $1 \mathrm{~s}$ (FEV1) of 35\% predicted $(1.00 \mathrm{~L})$ and FEV1/FVC ratio of 0.92 . At the time of anaesthesia induction for the spinal fusion, he was found to have severe trismus and limited neck extension, which led to traumatic intubation, difficulty securing the airway and cancellation of the planned procedure. He was admitted to the ICU, where chest $\mathrm{X}$-ray showed bilateral diffuse infiltrates, and he was noted to have bloody secretions from the endotracheal tube. He was intubated for 8 days and then transitioned to nocturnal NIPPV. The morning after extubation, while on NIPPV, the patient developed chest pain, and the chest X-ray revealed a moderate right-sided pneumothorax. A chest tube was placed and the patient eventually improved completely and was discharged on nocturnal NIPPV.

After discussion with the patient and family, a decision was then made to proceed with the previously planned spinal fusion after placement of a preemptive tracheostomy. In the immediate post-operative period, a small left-sided (contralateral) pneumothorax developed while the patient was treated with positive pressure ventilation via tracheostomy. A chest tube was required from post-operative day 1 to day 11 . 
After a prolonged hospital course, the tracheostomy was removed and the patient was discharged with nocturnal ventilation. He has not had a recurrence of pneumothorax to date.

\section{Cohort 2: adults with recurrent pneumothoraces}

Patient 4 is a 46-year-old female who was diagnosed at age 37 years with a heterozygous missense mutation in the COL6A1 gene and a clinical course consistent with the intermediate phenotype. Congenital onset symptoms included kyphoscoliosis and distal hyperlaxity. She walked at age 14 months and was diagnosed as unspecified congenital myopathy after two muscle biopsies. In her 20s and 30s, while still ambulant, she began to experience morning headaches. At age 35 years she was diagnosed with sleep apnoea by polysomnogram, but was intolerant of continuous positive airway pressure because of "claustrophobia". At age 37 she developed hypercapnic respiratory failure requiring intubation and ultimately tracheostomy. During hospitalisation, she developed her first right-sided pneumothorax, which was attributed to central line placement. She was eventually discharged home on chronic invasive mechanical ventilation for approximately $8 \mathrm{~h}$ per night.

At age 42 years she presented to the emergency department with her second pneumothorax. She described 3 days of left-sided pleuritic chest pain following a paroxysm of cough while on her home ventilator. On chest radiograph, she was found to have a moderate-sized left pneumothorax and a chest tube was placed at the bedside. Several hours later she developed frank haemoptysis, and bronchoscopy identified the source of bleeding to be the right lower lobe. A right-sided thoracentesis was also performed for effusion and $300 \mathrm{~mL}$ of blood was removed, confirming a right-sided spontaneous haemothorax in association with the pulmonary haemorrhage and left pneumothorax. Coagulation indices were normal and she required 4 units of packed red blood cells. A subsequent computed tomography (CT) scan showed a new right apical pneumothorax, diffuse patchy airspace disease and left lateral emphysematous changes (bullae). The small residual right pneumothorax did not require a chest tube and the effusions resolved over weeks. At the time of discharge she required a higher level of pressure support on her home ventilator, and daily ventilator use increased to $12 \mathrm{~h}$ per day.

Three months later, she developed pleuritic right chest pain after she leaned to the right during a transfer from wheelchair to car. Given prior challenges with chest tube insertion, the tube was placed by interventional radiology under fluoroscopic guidance. The pneumothorax resolved and the chest tube was removed. Two months later, she spontaneously developed her third right-sided pneumothorax.

A decision was made to proceed with video-assisted thoracic-surgical talc pleurodesis. During trochar insertion, the lung parenchyma was perforated due to multiple fibrous strands causing tenting of the lung to the chest wall. Lung tissue was described as abnormally fragile and friable, with extension of the existing laceration even under gentle pressure. To control blood loss $(800 \mathrm{~mL})$, the procedure was converted to an open thoracotomy. During the hospital stay and upon discharge, she could only tolerate tidal volumes of $375 \mathrm{~mL}$ (compared with prior volumes of $425 \mathrm{~mL}$ ). Ventilation was switched from pressure control to volume control, and her daily need for ventilator support increased to nearly $22 \mathrm{~h}$. She is now 46 years old and has not had any additional pneumothoraces since the VATS pleurodesis.

Patient 5 was a 26-year-old female when she first presented with a pneumothorax. She carried a lifelong diagnosis of "muscular dystrophy" and was specifically diagnosed with collagen VI-RD by skin biopsy at age 30 years with a heterozygous missense change in COL6A1. She initially presented with congenital weakness. She was able to crawl for a short time, but was unable to walk secondary to joint contractures. At age 6 years she underwent tendon transfers and was able to walk until age 12 years. She underwent scoliosis surgery at age 13 years. It was recommended that she use non-invasive ventilation (NIV) support at age 14 years, and regular nocturnal use began at age 18 years, with increasing daily use over time. Her phenotype was felt to be consistent with UCMD, given her loss of ambulation around age 10 years and the early need for NIV support.

She experienced her first pneumothorax at age 26 years, 3 days following a trial of mouthpiece ventilation administered via a volume-cycled ventilator in the pulmonary clinic. When she came to hospital she was found to have a $30-40 \%$ right-sided pneumothorax, attributed to the trial of volume ventilation. Unfortunately, chest tube thoracostomy revealed a large air leak, for which she required continuous suction and hospitalisation for over 2 months. A CT scan during that hospitalisation revealed bilateral subpleural blebs and some associated apical ground glass opacities. Given the severity of underlying lung disease and a baseline FEV1 of $13 \%$ predicted $(0.65 \mathrm{~L})$, a surgical approach was felt to carry an unacceptably high risk for death. For similar reasons, bedside pleurodesis was not performed. Ultimately, the air leak resolved, and she returned home using more frequent NIV for $14 \mathrm{~h}$ per day.

18 months later, she presented with left-sided chest pain 1 week following a minor motor vehicle accident. The chest X-ray was difficult to interpret due to severe kyphoscoliosis, which led to a delay in diagnosis, 

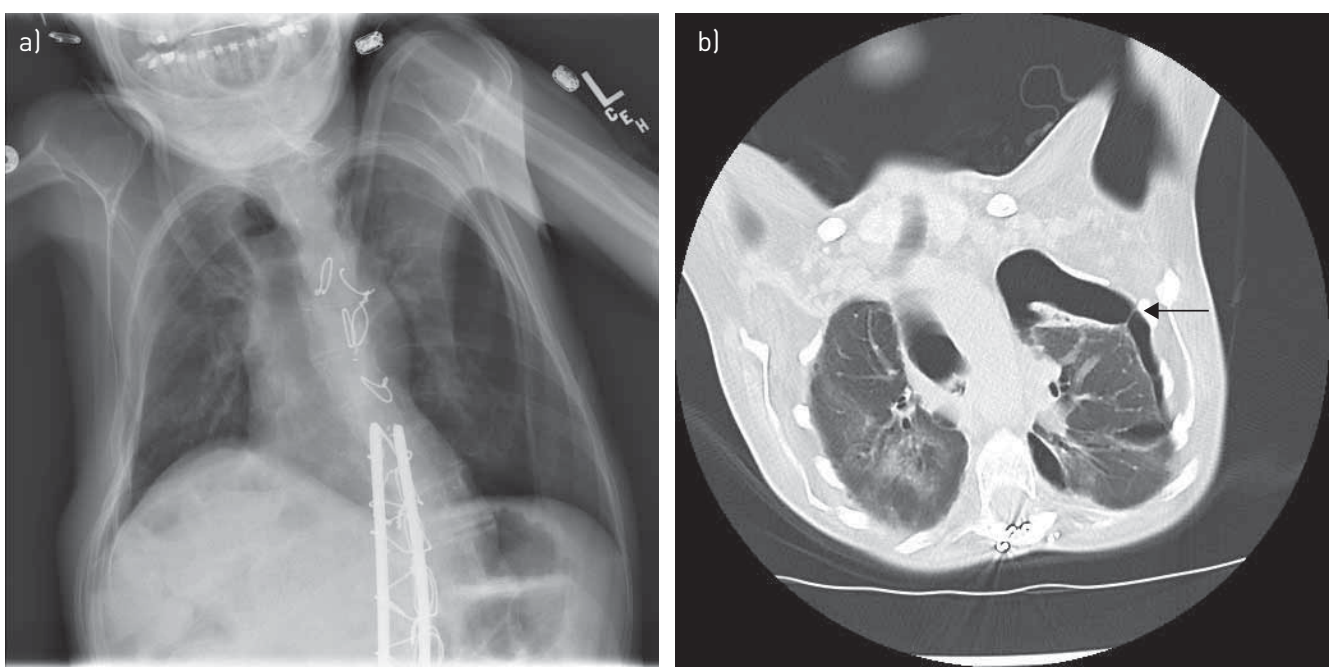

FIGURE 1 a) Chest X-ray and b) chest computed tomography on patient 5, presenting with a left pneumothorax, which is very difficult to appreciate on chest X-ray. Note the pleural adhesion (arrow) tethering the lung to the chest wall.

but ultimately left-sided (contralateral) pneumothorax was proven with a CT scan (figure 1a and b). With oxygen treatment, the pneumothorax resolved within several days but spontaneously recurred twice, on the left side, within the year.

For the next 4 years, she continued to have recurrent pneumothoraces, affecting both sides and requiring chest tube placement on seven subsequent occasions. Many of her episodes did not require intervention or hospitalisation because of the development of multiple adhesions within the pleural space (figure 1b). Similar to patient 4 , she perceived that torso movement, particularly twisting, was a trigger for successive pneumothoraces. On one occasion her only presenting symptom was right-sided swelling under her arm, due to spontaneous subcutaneous emphysema and small pneumothorax (figure 2).

Repeat CT scans over 4 years documented persistent and stable patchy ground-glass opacities bilaterally in the lung apices, associated with subpleural blebs (figure 3a and b). A 3-month trial of oral corticosteroids for the parenchymal opacities (for possible cryptogenic-organising pneumonia) had no effect on the symptoms or radiographs. Despite near-24 h NIV, she declined tracheostomy and her respiratory failure progressed. Unfortunately, she passed away at age 32 years from community-acquired pneumonia.

\section{Discussion}

In this case series (table 1) we describe five patients with confirmed collagen VI-RD who developed pneumothoraces: three were children with isolated events related to medical procedures and two were young adults who developed recurrent pneumothoraces without traditional inciting barotrauma. In each case, the clinicians attributed the pneumothorax to an associated stressor, including vaginal delivery,

FIGURE 2 Chest computed tomography of patient 5 , presenting with right arm swelling due to small apical pneumothorax and subcutaneous emphysema, treated conservatively with supplemental oxygen therapy.

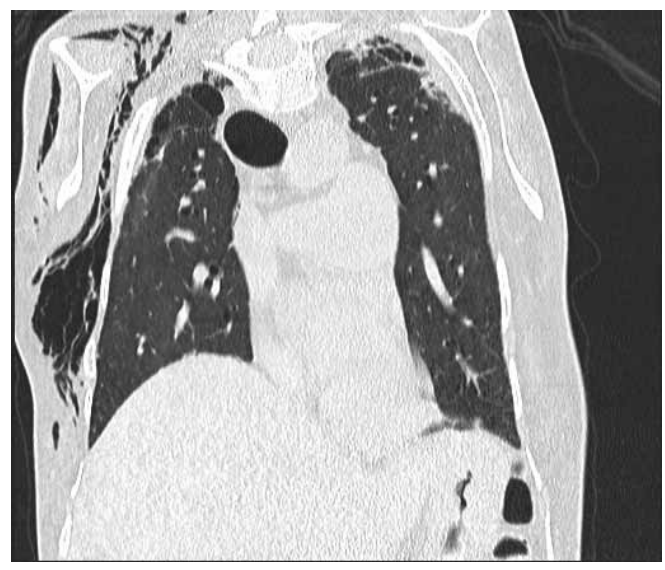


resuscitation efforts, central line insertion, spinal surgery, non-invasive mouthpiece ventilation, twisting of the torso during patient transfer and minor motor vehicle accidents. However, the frequency of these pneumothoraces and the associated pleural/parenchymal changes suggest that pneumothoraces are an important and previously under-recognised manifestation of collagen VI-RD.

From the literature we were able to identify one further patient who fits the clinical phenotype of collagen VI-RD with pneumothorax. He was initially described in 2002 [5] prior to the availability of molecular confirmation for this disorder. He presented with congenital hip dislocation, distal hyperlaxity, contractures and, at age 21 years, he required nocturnal NIV. At age 26 years, he presented with chest pain 4 days after a motor vehicle accident and was diagnosed with a small apical pneumothorax. His chest CT scan revealed multiple blebs. The patient went on to have three recurrent left-sided pneumothoraces, which were treated, respectively, with tube thoracostomy, doxycycline chemical pleurodesis through a chest tube, and an open thoracotomy for pleurodesis. At surgery, multiple fibrinous strands were described to be tenting the lung to the chest wall with surface blebs. Unfortunately, there is no further follow-up available on this patient. In the initial report, the authors hypothesised that patients with disorders of collagen type VI might be predisposed to the development of pleural blebs.

Collagen VI is a key structural component of the pulmonary extracellular matrix, playing a developmental role in lung embryogenesis and in the maintenance of lung parenchyma [6]. Limited immunofluorescent work in human lungs has shown collagen VI is present in the walls of bronchi and bronchioles and in pulmonary vessels [4]. Staining for collagen VI in the epidermal-dermal junction and lung alveoli using co-localisation with laminin antibodies confirms the role of collagen VI in maintaining a tight connection between the lung extracellular matrix and basement membrane [7].

Given the abnormal appearance of the lung parenchyma on CT scans in two of our patients and one from the literature, we suspect that patients with collagen VI-RD are more prone to spontaneous and barotrauma-related pneumothoraces because of a primary lung parenchymal defect. While patients 4 and 5 both developed pneumothoraces after initiation of nocturnal ventilation, not all events in our adult patients occurred while on their positive pressure devices. Interestingly, both patients 4 and 5 ascribed pneumothoraces to "twisting" during a patient transfer manoeuver, which is not a typical trigger for pneumothorax in other conditions. Similarly, one case of pneumothorax was attributed to a minor motor vehicle accident that occurred several days prior to the onset of symptoms, timing that is not typical for

TABLE 1 Clinical and genetic phenotype of collagen VI-related dystrophy patients with pneumothorax (PTX)

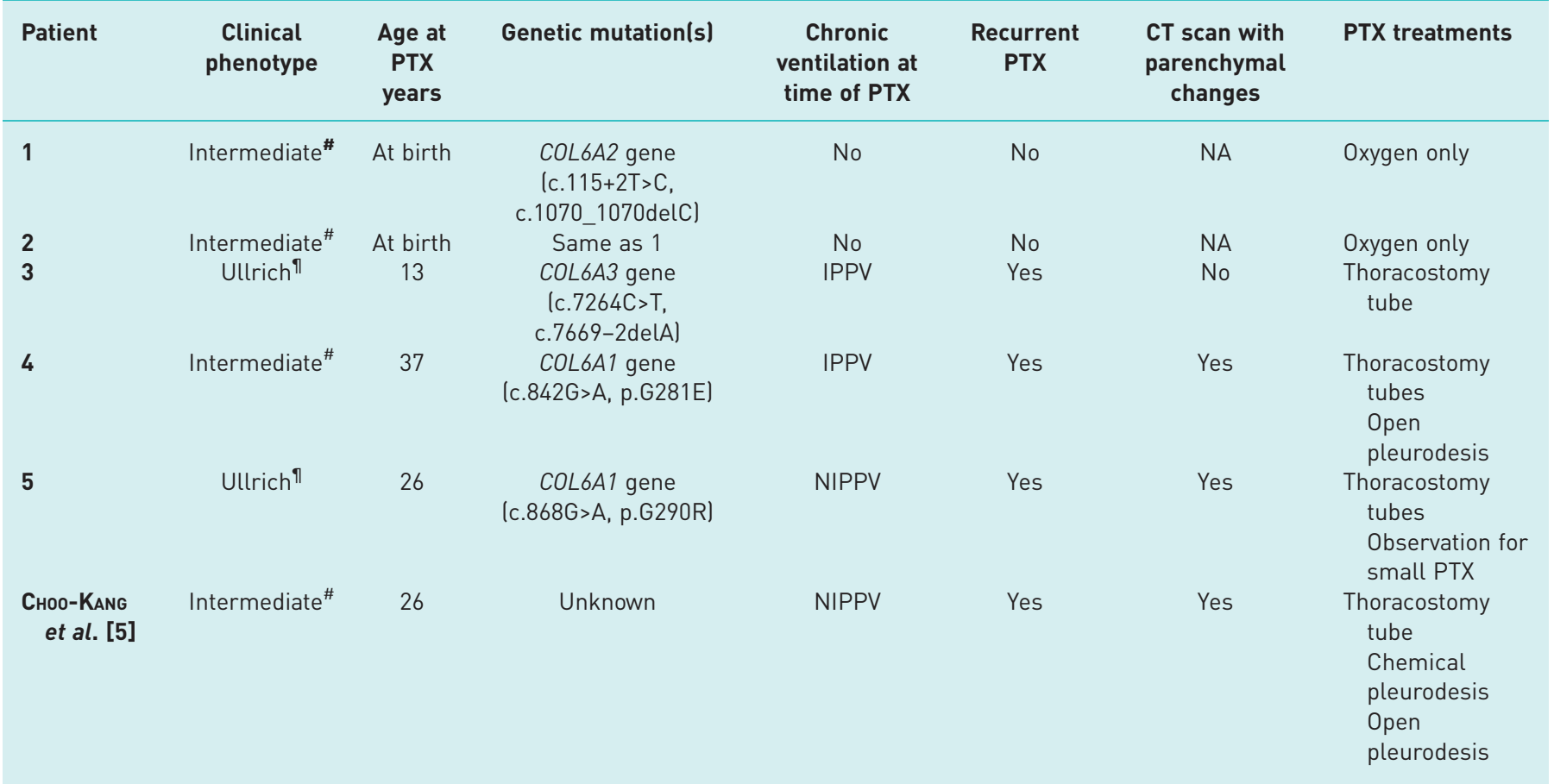

CT: computed tomography. IPPV: invasive positive pressure ventilation; NIPPV: noninvasive positive pressure ventilation; NA: not available. \#: ambulant until adulthood with progressive respiratory insufficiency; ${ }^{\text {I: }}$ ambulation never achieved or lost prior to age 10 years. 

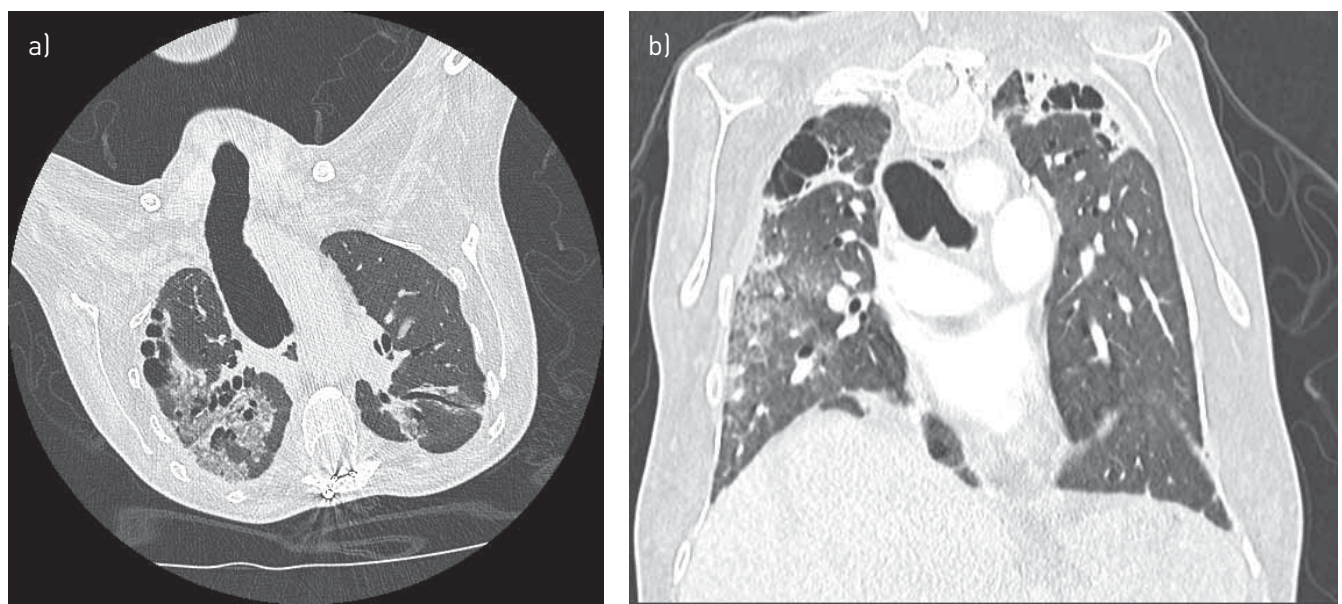

FIGURE 3 Computed tomography scans of patient 5 showing a) axial and b) coronal views of diffuse parenchymal lung disease with ground-glass opacities and apical bullae.

traumatic pneumothorax. While there have been case reports suggesting that spontaneous pneumothorax in muscular dystrophy might be due to NIV [8], one retrospective single-centre study of inpatients with muscular dystrophy (without the availability of molecular subtyping in 1994) reported an 18\% risk of pneumothorax, but they could not show an increased risk in those using mechanical ventilation [9].

Knowledge gained from studying the molecular pathogenesis of the CMDs highlights genetic subtype-specific differences in disease expression and pathophysiology [10]. Many young adults with CMD graduate from their paediatric neurologists to adult practitioners, who need to be aware that identifying the specific subtype of muscular dystrophy may better predict the clinical course and guide pulmonary and clinical surveillance. The importance of genetic/molecular subtyping of patients with muscular dystrophy is underscored by this case series. Any muscular dystrophy patient with pneumothorax should probably be assessed for potential parenchymal lung disease before assigning full causation to triggers such as the use of NIV. Importantly, NIV is life-prolonging therapy and should not be withheld in the event of pneumothorax; rather, we would recommend aggressive definitive treatment of pneumothorax in any patient with collagen VI-RD.

From this case series we identify several potential management issues for patients known to have collagen VI-RD.

\section{Difficulties with diagnosing pneumothorax}

Body habitus distortions in collagen VI-RD, including kyphoscoliosis and pectoralis contractures, can make it difficult to identify pneumothorax by physical examination and/or routine chest radiographs (figure 1a and b). Practitioners should have a low threshold for ordering a chest CT when the history suggests pneumothorax.

\section{Chest tube insertion is challenging}

Weak and elevated diaphragms, kyphoscoliosis, muscle contractures and downward angled ribs can make it difficult to properly position patients and to identify appropriate landmarks for chest tube insertion. Such factors might have contributed to the misplacement of the chest tube by an experienced surgeon and subsequent complications in our patient 4. Placement of the chest tube under image guidance will reduce the risk of such complications, including inadvertent tube insertion into lung parenchyma if pleural space adhesions are present.

\section{Pleural adhesions may affect presentation}

None of the patients with spontaneous pneumothoraces developed tension pneumothorax even when on positive pressure ventilation. Surgical intervention in one case described multiple fibrous strands between the visceral and the parietal pleura, a finding also visualised on CT imaging of patient 5 (figure 1b). Such adhesions could potentially tether the lung to the chest wall and thus reduce the development of a tension pneumothorax, an effect described as "auto-pleurodesis". In fact, both patients 4 and 5 experienced occasions of small pneumothoraces that remained stable and were able to be treated conservatively without tube thoracostomy. 
We recommend early definitive therapy for patients with advanced lung disease

An open approach to pleurodesis may be required given the presence of the fibrous strands and a higher risk of perforating lung parenchyma with less invasive approaches. However, patients with advanced respiratory failure are at high risk of post-operative complications and patient 5 was not a surgical candidate by the time that this intervention was considered. Knowing the high likelihood of recurrent events in this population should prompt the practitioner to consider early surgical consultation for effective intervention. Inevitably, these patients will require increases in the intensity or duration of ventilation due to respiratory muscle failure and chest wall rigidity. As ventilation requirements increase, the risk for pneumothorax will also increase, underscoring the importance of early definitive therapy.

\section{Titrate NIV to minimise peak airway pressure}

If a patient with pneumothorax is dependent on NIV, then pressure-limited ventilation (bilevel positive airway pressure) is preferable to volume cycled modes for minimising peak airway pressure and intrathoracic pressure swings [11]. Extending the inspiratory time to achieve the desired volume at a lower pressure can be quite effective, and many of the newer bilevel ventilators have multiple options for optimising ventilation, including volume-targeted approaches. Clinicians treating neuromuscular patients require expertise in bedside and polysomnographic titration of NIV, the interpretation of downloaded data and strategies for improving patient-ventilator synchrony for achieving optimal compliance.

\section{Conclusion}

Collagen VI-RD is characterised by progressive restrictive respiratory impairment. Pneumothoraces can contribute to further restriction from worsening pulmonary compliance. Paradoxically, practitioners concerned about the possibility of recurrent pneumothoraces might try to limit ventilator pressures, but this can further reduce chest wall compliance and worsen the associated hypoventilation. Having described this group of patients with a high risk of pneumothoraces, we feel that additional studies are needed to determine the prevalence of parenchymal lung disease and pneumothorax in patients with collagen VI-RD. We also recommend investigation into the parenchymal changes in the varied clinical phenotypes of collagen VI-RD, potential triggers for pneumothorax across the lifespan of these patients, and consideration of screening for potential early intervention, prior to the initiation of long-term positive pressure ventilation.

\section{Acknowledgements}

The authors would like to thank the patients for their stories, the Congenital Muscle Disease International Registry staff for record review and Ying $\mathrm{Hu}$ at the National Institutes of Health for molecular diagnostics.

\section{References}

Bönnemann CG. The collagen VI-related myopathies: muscle meets its matrix. Nat Rev Neurol 2011; 7: 379-390.

2 Quijano-Roy S, Khirani S, Colella M, et al. Diaphragmatic dysfunction in collagen VI myopathies. Neuromuscul Disord 24: 125-133.

3 Foley AR, Quijano-Roy S, Collins J, et al. Natural history of pulmonary function in collagen VI-related myopathies. Brain 2013; 136: 3625-3633.

4 Godoy-Guzmán C, San Martin S, Pereda J. Proteoglycan and collagen expression during human air conducting system development. Eur J Histochem 2012; 56: e29.

5 Choo-Kang LR, Ogunlesi FO, McGrath-Morrow SA, et al. Recurrent pneumothoraces associated with nocturnal noninvasive ventilation in a patient with muscular dystrophy. Pediatr Pulmonol 2002; 34: 73-78.

6 Kuo HU, Maslen CL, Keene KR, et al. Type VI collagen anchors endothelial basement membranes by interacting with type IV collagen. J Biol Chem 1997; 272: 26522-26529.

7 Belton JC, Crise N, McLaughlin RF, et al. Ultrastructural alterations in collagen associated with microscopic foci of human emphysema. Hum Pathol 1977; 8: 669-677.

8 Vianello A, Arcaro G, Gallan F, et al. Pneumothorax associated with long-term non-invasive positive pressure ventilation in Duchenne muscular dystrophy. Neuromuscul Disord 2004; 14: 353-355.

9 Yamamoto T, Kawai M. Spontaneous pneumothorax in Duchenne muscular dystrophy. Clin Neurol 1994; 34: $552-556$.

10 Wang $\mathrm{CH}$, Bönnemann CG, Rutkowski A, et al. Consensus statement on standard of care for congenital muscular dystrophies. J Child Neurol 2010; 25: 1559-1581.

11 Simonds AK. Pneumothorax: an important complication of non-invasive ventilation in neuromuscular disease. Neuromuscul Disord 2004; 14: 351-352. 